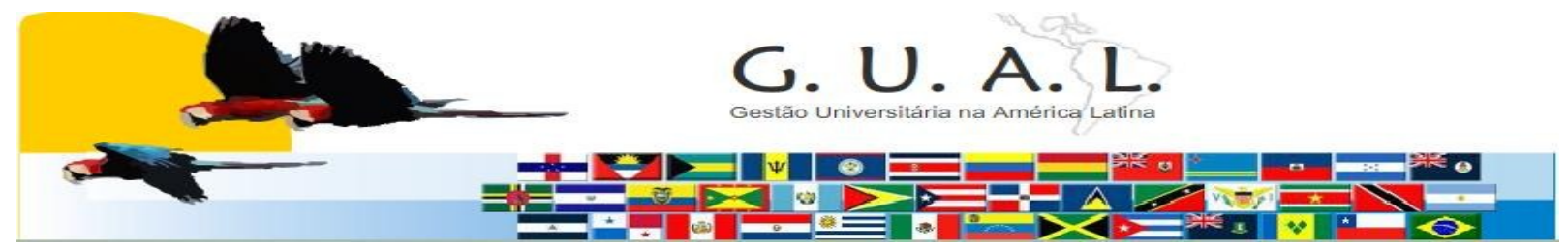

ISSN 1983-4535

\title{
MOVIENDO VOLUNTADES: LOS AGENTES DEL CAMBIO Y LA ORGANIZACIÓN UNIVERSITARIA INTERAMERICANA
}

\author{
Maria del Pilar Velasco Muñoz-Ledo, Mestre \\ Universidad Veracruzana - México \\ IGLU. OUI. \\ mvelasco@uv.mx
}

\begin{abstract}
RESÚMEN
Durante los últimos años, la educación superior se ha enfrentado a uno de los retos más serios de las últimas décadas, que impone limitaciones y desafíos específicos para el establecimiento de su agenda a nivel mundial. Las instituciones de educación superior (IES) afrontan demandas y presiones para cumplir su función social ante una sociedad que se transforma drásticamente y que enfrenta, a su vez, problemáticas y retos emergentes que requieren nuevos desarrollos científicos, sociales y profesionales más acordes a las necesidades sociales. Pero sería un error pensar que la transformación de la educación superior descansa únicamente en la claridad de sus propósitos con miras al futuro: es necesario también el desarrollo de los agentes del cambio que la llevarán a cabo. Los académicos son, sin duda, actores fundamentales del diseño y preparación de esta evolución, pero replantear las funciones de investigación, formación y vinculación implica igualmente un cambio en las formas de organización y gestión; así, los líderes y administradores de las IES deben prepararse para anticipar y conducir los cambios que lograrán mejores instituciones con una mayor contribución social. La Organización Universitaria Interamericana pretende contribuir en la formación de tales agentes, a través del IGLU y los servicios que éste proporciona a las instituciones afiliadas.
\end{abstract}

Palabras claves: Voluntades. Agentes. Universidad. 


\section{EL CONTEXTO}

A nivel mundial, la educación superior inició el siglo XXI enfrentando profundas transformaciones que tienen grandes repercusiones en los ámbitos económico, social, político, cultural: la matrícula y la cobertura crecen; la oferta educativa se consolida y se amplía; la calidad de la educación pasa de ser aspiración y valor compartido, a necesidad para la subsistencia de las IES.

Las universidades, en particular las de América Latina, enfrentan un reto multidimensional que abarca desde el aseguramiento de los recursos financieros, en condiciones de franca restricción presupuestal (al tiempo que hay un crecimiento sustancial del estudiantado disminuye el gasto público destinado a la educación superior), hasta la ampliación de la cobertura a largo plazo, el fortalecimiento de la calidad del proceso enseñanza-aprendizaje, el cierre de brechas, entre otros.

Sin duda la sociedad actual tiene enormes expectativas respecto a la generación y aplicación del conocimiento, y en cuanto a la formación de técnicos, profesionales y científicos cada vez más competentes. Una educación superior pertinente y de calidad no es únicamente una aspiración legítima, sino una condición indispensable y fundamental para impulsar el desarrollo de nuestros países, mejorar los niveles de competitividad, coadyuvar a una inserción más ventajosa en la economía mundial y encontrar alternativas eficientes para enfrentar los problemas que afectan a la humanidad. ${ }^{1}$

Si estamos de acuerdo en que la misión fundamental de las IES es la de crear y transferir el conocimiento en favor de la sociedad, la universidad debería constituirse (como lo menciona Fernández Lamarra) en el "ámbito principal de creatividad e innovación para sí misma y para la sociedad en su conjunto,"2 por lo que uno de los retos fundamentales a los que se enfrenta es el de generar conocimiento que impacte los sectores productivos y contribuya a la solución de los problemas sociales. La educación superior se constituye en la mejor vía para la transformación y desarrollo de nuestros países, y es responsabilidad de las universidades el adaptarse a los cambios que la sociedad le exige.

En la llamada "tercera revolución" del conocimiento, uno de los retos significativos de todas las universidades - independientemente de su contexto geográfico - es el de generar fuentes que impacten en los sectores productivos y, por ende, contribuyan a la solución de problemas sociales; se trata de utilizar el conocimiento para beneficiar a la sociedad en su 


\section{MOVIENDO VOLUNTADES: LOS AGENTES DEL CAMBIO Y LA ORGANIZACIÓN UNIVERSITARIA INTERAMERICANA}

conjunto y, por lo tanto, son las propias universidades las que deben generar "ambientes que potencien las relaciones entre los universitarios y los sectores productivos". ${ }^{3}$

Las universidades enfrentan retos de distinta naturaleza y complejidad. Las instituciones deben adaptarse a la velocidad de los cambios y deben transformar sus modelos pedagógicos haciendo uso intensivo de las nuevas tecnologías de información y comunicación (TIC); los desafíos implican cambios en el currículum y en las modalidades de enseñanza (con diseños innovadores que atiendan los requerimientos sociales y académicos); mayor flexibilidad y mayor cobertura (sin menoscabo de la calidad y la pertinencia de los programas académicos); con perspectiva de futuro; con enfoque de educación permanente (para la vida).

La Universidad no es únicamente un espacio de mera "instrucción" en el que se forman personas competentes para el desarrollo social y económico, como promueve la OCDE, sino el ámbito en el que se forman ciudadanos: la educación en gran medida forma el carácter moral de seres humanos.

Los serios problemas políticos, económicos, de planificación, plantean evidentemente importantes desafíos para la gestión. La formación de los universitarios del siglo XXI exige, como ya se ha mencionado, la excelencia académica; la eficiencia; la capacidad de adaptación a los constantes cambios sociales y políticos; el conocimiento de las políticas de educación superior (a nivel internacional, regional y local); capacidad política; liderazgo y destreza para unir voluntades y para traducir y satisfacer las necesidades integrales. Y todo ello obliga a una profunda revisión de las estructuras y las formas de gobierno, a fin de responder de manera cada vez más apropiada al contexto dinámico de la sociedad, y exige mejores resultados organizacionales y el desarrollo de una más amplia capacidad en los procesos de gestión. Es nuestra responsabilidad el formar universitarios capaces de comprender los complejos desafíos, con una visión de largo alcance, y de promover innovaciones que conduzcan a la comunidad universitaria hacia soluciones y toma de decisiones novedosas, siempre en interés de la institución y la sociedad. conocer y dominar los conceptos y técnicas de gestión de las organizaciones.

Pero más aún, es también responsabilidad de las instituciones de educación superior el formar universitarios capaces de conocer y dominar los conceptos y técnicas de gestión de las organizaciones, particularmente las educativas; y de atender los problemas universitarios con una perspectiva de futuro, y de encontrar los caminos más apropiados que muevan las 
voluntades, que convoquen y motiven a la comunidad universitaria hacia soluciones y toma de decisiones acordes con las cambiantes necesidades sociales.

\section{LA ORGANIZACIÓN UNIVERSITARIA INTERAMERICANA}

A partir de su fundación en 1979, la Organización Universitaria Interamericana (OUI) ha constituido un espacio fundamental para el desarrollo de la educación superior de las Américas, consolidándose como foro de discusión y acuerdos sobre la agenda de este nivel educativo. Desde sus inicios, la OUI ha sustentado su actividad bajo la perspectiva de que el verdadero papel de la universidad es, de hecho, un papel social; en palabras de su Presidente fundador, Gilles Boulet: "Existe en cada comunidad, en cada nación, en cada país, un motor indispensable para el mejoramiento social, y ese motor es la Universidad.”

A lo largo de su existencia, la OUI ha promovido y facilitado servicios, y ha coadyuvado a la reflexión sobre el papel que tienen o debieran tener los académicos, los estudiantes, los acervos bibliográficos, las nuevas tecnologías y las propias instituciones, en los procesos que facilitan el aprendizaje y hacen más efectivas y relevantes las enseñanzas. Su Misión: "fortalecer la contribución de la educación superior al desarrollo sustentable y a una mejor comprensión intercultural e integración de los pueblos de las Américas, por medio de la consolidación de sus programas, construyendo, renovando e innovando espacios comunes de cooperación interuniversitaria entre sus miembros y articulándolos con otros aliados estratégicos, en marcos colaborativos con otras regiones". 4

El Programa de Trabajo 2009-2011 y el Plan de Acción 2011-2016 renuevan el compromiso de continuar la reflexión sobre el papel académico y social, la organización y la gestión de las instituciones de educación superior de las Américas; pero al mismo tiempo, destacan la contribución sustancial de la educación superior en el proceso de integración concertada del continente y la protección de las identidades culturales, y refrendan sus responsabilidades en tanto instrumento de formación, intercambio y reflexión en el campos de la gestión universitaria; de liderazgo de diversas redes de formación (que agrupan a académicos que colaboran en el estudio y solución de problemas de interés hemisférico, y que diseñan cursos insertables en los programas académicos universitarios); de promoción del uso de las tecnologías como medio para intensificar la colaboración académica, la comprensión 


\section{MOVIENDO VOLUNTADES: LOS AGENTES DEL CAMBIO Y LA ORGANIZACIÓN UNIVERSITARIA INTERAMERICANA}

intercultural, las estrategias de ayuda mutua y cooperación, en el contexto de la diversidad cultural de sus miembros.

La OUI ha retomado pues, los desafíos en los que considera que la colaboración entre instituciones, organizaciones, países y regiones -en perspectiva de gran escala- puede hacer una diferencia para la educación superior de las Américas.

Tales retos contemplan la renovación de la visión que se tiene desde el continente americano, en donde es necesario redefinir el papel de las universidades para generar más conocimiento y para crear condiciones que contribuyan a su distribución social real; el propósito de la existencia de las IES, desde esta perspectiva, "no puede ser otro que el de colaborar a construir una sociedad del conocimiento vinculada con los problemas reales de las naciones; una sociedad con capacidad para generar, apropiarse y utilizar ese conocimiento para atender las necesidades de su desarrollo y así construir su propio futuro". 5

Son seis los ejes estratégicos que la Organización Universitaria Interamericana ha concebido para realizar su actividad en el próximo lustro: Innovación en sus programas para la cooperación y formación de capital humano de las IES; Innovación para la vinculación y el desarrollo; Espacios comunes de educación superior; Internacionalización; Gobernabilidad; Visibilidad (éstos dos últimos, considerados de apoyo). Los objetivos estratégicos que se derivan, y las acciones para alcanzarlos, se desarrollan a través de los tres programas de la OUI: IGLU, COLAM y CAMPUS.

El IGLU (Instituto de Gestión y Liderazgo Universitario) es el primer programa creado por la OUI (1983). Su finalidad es la de apoyar a las instituciones asociadas en los procesos formación, mejoramiento y consolidación de sus equipos humanos dedicados a la dirección académica y administrativa, y ha contribuido al desarrollo institucional de las universidades utilizando diversas intervenciones de cambio y aprendizaje, tanto organizacional como profesional, privilegiando el humanismo, la calidad y la pertinencia, así como la colaboración interinstitucional. Desde hace casi 30 años ha apoyado la formación de los dirigentes y directivos universitarios de América Latina y el Caribe y, por lo tanto, ha favorecido la pertinencia de las IES, la inserción de éstas a su entorno, y su transformación como órgano motor del desarrollo sostenible.

El COLAM (Colegio de las Américas) se constituye en 1997 como espacio promotor de la cooperación entre las universidades del continente americano, a través de la promoción y 


\section{MOVIENDO VOLUNTADES: LOS AGENTES DEL CAMBIO Y LA ORGANIZACIÓN UNIVERSITARIA INTERAMERICANA}

creación de consorcios académicos (Redes Interamericanas de Formación), en apoyo a la investigación en temáticas prioritarias de las IES, y en aquéllas que son demandadas por la sociedad, con una perspectiva interdisciplinaria, intercultural e innovadora.

Actualmente el COLAM cuenta con una decena de Redes en temas tan variados como los determinantes sociales de la salud, mujeres y desarrollo equitativo, educación y telemática, y otras; su estrategia se ha basado en la educación a distancia y en el uso de las tecnologías de información y comunicación. Actualmente cuenta con más de una

Una década después, la OUI vuelve a plantearse la necesidad de contar con un nuevo programa que canalice las propias fortalezas institucionales y las de sus miembros, hacia la reflexión sobre las temáticas centrales del mundo universitario, la formación en valores y el análisis crítico de las tendencias mundiales de la educación superior, y su aplicación concreta en nuestras universidades. CAMPUS apoya la construcción de un espacio de educación superior interamericano, a través de nuevos instrumentos de gestión del conocimiento, y de la discusión, análisis y desarrollo sobre temas estratégicos y nuevas tendencias de la educación superior. Entre sus líneas de acción se contempla un espacio de movilidad (intercampus) y otro de investigación e innovación (doctorados de tiempo compartido e incubadoras de investigación).

En la actualidad, la OUI agrupa a más de 300 instituciones de educación superior con una matrícula superior a los 5 millones de estudiantes, atendida por más de 500,000 académicos; sus miembros se localizan en 26 países organizados en 9 regiones: América Central, Brasil, Canadá, Caribe, Colombia, Cono Sur, Estados Unidos, México y Países Andinos.

\subsection{El nuevo Programa IGLU}

Como instrumento de formación, intercambio y reflexión en el campo de la gestión universitaria, el Instituto de Gestión y Liderazgo Universitario ha apoyado la capacitación de más de 1,500 dirigentes mediante el "Curso IGLU" y de casi 20,000 en sus diversos Seminarios. Con el soporte de instituciones y profesionales de la educación superior, ofrece productos y servicios relevantes para la gestión educativa y el liderazgo universitario en seis Coordinaciones y/o Centros Regionales que se alojan en Universidades colaboradoras de cada región:

Rev. GUAL., Florianópolis, v.4, n. 1, p.70-81, jan/abr. 2011 
- México: Universidad de Guanajuato;

- América Central: Universidad de Costa Rica;

- Caribe: Universidad Simón Bolívar (Venezuela);

- Países Andinos: Universidad Cayetano Heredia (Perú);

- Brasil: Universidad Federal de Santa Catarina;

- Como Sur: Universidad Nacional de La Matanza (Argentina).

En sus casi tres décadas de existencia, el curso ha ido evolucionando a fin de dar respuesta a las cambiantes condiciones y los retos que la educación superior de las Américas han ido planteándose, y su proceso de transformación continúa con las valiosas aportaciones de quienes han recibido formación en sus aulas, la mayor parte de los cuales continúan hoy en funciones directivas, ya sea en sus instituciones de origen o en entidades y organizaciones relacionadas con la gestión educativa, y de líderes educativos especialistas en los temas de vanguardia.

A partir de la reflexión sobre las circunstancias cambiantes del entorno y los escenarios que enfrenta la educación superior, el IGLU inició en el 2009 un proceso de renovación que le permitirá mantener su vigencia en el contexto actual e incrementar la eficacia de sus actividades. El ejercicio ha incluido la deliberación sobre las características que tendrán las instituciones de educación superior en los próximos años, y los directivos que requerirán para alcanzar sus objetivos; la búsqueda de una mayor inter-americanidad del Diplomado o "Curso IGLU" y de los otros servicios (cursos cortos, talleres, seminarios); la apertura de nuevas posibilidades de apoyo a la gestión (como el establecimiento de pasantías o estancias especializadas); entre otros aspectos. Los ejes estratégicos que articulan las actividades del Instituto de Gestión y Liderazgo Universitario de la Universidad Veracruzana (UV) y la Organización Universitaria Interamericana (OUI) en su nuevo programa, permitirán fortalecer la gestión institucional de las universidades e incentivar su internacionalización, al ofrecer una cartera de servicios diversificada y actual, acorde con las necesidades regionales.

En este marco, destacan las actividades tendientes a la identificación de ejes temáticos de interés común para los miembros de la organización; la apertura de espacios de consulta que permitan identificar las necesidades de formación de su personal académicoadministrativo, y que resultan prioritarias para la eficaz gestión institucional; la búsqueda de 
alianzas estratégicas para establecer una Red de Posgrados en gestión educativa, identificando espacios de complementariedad académica y la Red Interamericana de Mejoramiento de la Gestión Universitaria; y la promoción de herramientas de gestión que impulsen el acceso y faciliten la consulta de información bibliográfica y de bases de datos en línea (como el Observatorio en gestión universitaria, la Biblioteca Virtual y la Red de Repositorios Virtuales).

Para alcanzar los objetivos planteados se han instrumentado diversas acciones, entre la que destaca la realización de la "Conferencia de Búsqueda para la innovación de la formación de líderes y administradores de instituciones de educación superior”, con la participación de los responsables y actores principales de programas de formación de todo el Continente Americano, ya sea que se ofrezcan por parte de organizaciones o de instituciones de educación superior. ${ }^{6}$

El encuentro se llevó a cabo el pasado mes de febrero en la Universidad Veracruzana, con la participación de más de 30 directivos universitarios y/o especialistas en gestión educativa de todo el Continente. ${ }^{7}$ Bajo la coordinación de expertos en estas técnicas, discutieron temas tales como la universidad del futuro; la responsabilidad social de las Universidades; los retos de la colaboración interamericana y, por supuesto, el "perfil" del administrador universitario ideal, y de las cualidades y competencias que resultan indispensables para el desarrollo de sus actividades.

Al plantear un horizonte a largo plazo, y analizar las nuevas dinámicas que impone la producción de conocimiento, se esbozaron algunos de los principales desafíos que tendrán las universidades en los próximos años, entre otros:

- una oferta de formación diversificada que permita atender poblaciones estudiantiles variadas y con nuevas necesidades;

- la necesidad de trabajo inter y transdisciplinar;

- un incremento en la movilidad (de alumnos, de académicos, de administradores);

- una mayor vinculación con la sociedad, el gobierno, la empresa;

- mayores restricciones presupuestales y, por lo tanto, la necesidad de encontrar fuentes de financiamiento alternas;

Rev. GUAL., Florianópolis, v.4, n. 1, p.70-81, jan/abr. 2011 
- una mayor presión social por el ingreso a la educación superior y, al mismo tiempo, mayores índices de desempleo;

- el establecimiento programas educativos que respondan a las necesidades sociales y que permitan ampliar la cobertura de la educación superior;

- innovación creciente que permita la revalorización e intensificación de la comunicación entre los nuevos actores y la creación de nuevos espacios para el aprendizaje;

- mejores sistemas de apoyos económicos para los estudiantes (fortalecimiento del sistema de becas); etc.

El nuevo escenario en el que se replantean las relaciones sociedad-ciencia y se generan las bases de un espacio común de la educación y la cultura, se vislumbran retos que no son menores, dado que se hace necesaria la transformación y flexibilización en la estructura organizativa y se requieren cambios en el perfil de los líderes y gestores universitarios que trazarán el rumbo y la estrategia del desarrollo institucional, al tiempo que comprenden las nuevas demandas que plantea la sociedad.

Los nuevos dirigentes universitarios deberán ser capaces de asumir también el liderazgo social en la creación de conocimiento para enfrentar los complejos desafíos mundiales: seguridad alimentaria, cambio climático, gestión del agua, energías renovables, salud pública, interculturalidad, etc.

En este camino se presentan dilemas importantes; para el caso del currículo universitario, por ejemplo, Mora $^{8}$ señala algunos: atender las necesidades del mercado o las de la sociedad en su conjunto; formar profesionales o ciudadanos profesionales; enfatizar en los contenidos o en los contenidos, las destrezas, las habilidades y los valores; acentuar la enseñanza o los aprendizajes; comprender y comprometerse con los problemas locales y regionales, o con la dimensión global. En suma, lo que llama el contrapunto entre la dimensión individual, el ámbito social y colectivo y el desarrollo humano integral

Indudablemente, el dirigente y directivo universitario debe contar con los conocimientos y herramientas de gestión (especialmente de planificación, de gestión de recursos y de valoración de las TIC); debe estar al tanto de las políticas de educación superior a nivel nacional, regional e internacional, a fin de estar en posibilidades de desarrollar las reformas educativas necesarias para la competitividad; debe ser capaz de establecer diálogos, 
consensos y negociaciones; pero más aún, debe ejercer su liderazgo con compromiso ético de servicio, colaboración y promoción de los valores académicos.

Hoy, más que nunca, la educación superior es la herramienta más poderosa para contender con el futuro, con el desarrollo, y con las incertidumbres que la crisis ha abierto, y se ha vuelto imprescindible el realizar un esfuerzo especial para crear las condiciones que permitan alcanzar una real distribución social del conocimiento, a efecto de hacer de éste una palanca efectiva del desarrollo.

A través de acciones conjuntas interinstitucionales es posible potenciar a los líderes y administradores de las IES, pero para ello es necesario pensar y trabajar de forma colaborativa entre representantes de las diferentes regiones americanas los aspectos prioritarios que los programas de formación y actualización deberán tener en los próximos años. Las prioridades compartidas a nivel interamericano darán lugar a definir un núcleo básico común que les de identidad y unidad a los programas de formación en las distintas regiones de América y, al mismo tiempo, permitirán identificar las necesidades de formación específicas de las regiones que deberán ser incorporadas en estos programas.

El nuevo programa de formación del IGLU que se propone, toma en consideración estos desafíos y promueve la participación de especialistas en el diseño de los contenidos de un tronco común interamericano, y de la nueva oferta académica pertinente, que incorpore las nuevas temáticas con un enfoque de competencias, metodologías pedagógicas adecuadas o con diferentes modalidades.

\section{REFERENCIAS}

\footnotetext{
${ }^{1}$ La producción y la acumulación de conocimientos son actualmente la fuerza fundamental en el contexto de la globalización, de acuerdo a la Organización de las Naciones Unidas para la Educación, la Ciencia y la Cultura (UNESCO) y la Comisión Económica para América Latina y el Caribe (CEPAL).

2 Fernández Lamarra, N. (2010). Hacia una nueva agenda de la educación superior en América Latina. ANUIES. Colección Temas de Hoy en la Educación Superior No. 028. México, D.F.

3 Arias Lovillo, R. "La formación de líderes y gestores universitarios de excelencia". UniVerso. Universidad Veracruzana. México. Año 10, No. 430. 14 de febrero, 2011.
}

Rev. GUAL., Florianópolis, v.4, n. 1, p.70-81, jan/abr. 2011 
${ }^{4}$ Documento OUI. Orientaciones estratégicas 2011-2016.

${ }^{5}$ Ibídem.

${ }^{6} \mathrm{La}$ "Conferencia de Búsqueda" es una técnica muy empleada para encontrar acuerdos sobre los elementos básicos que perfilan la planeación estratégica, en situaciones cambiantes o poco definidas (Malo, Salvador. 2011)

${ }^{7}$ Rectores, Vice-rectores, Decanos, Directores Generales y otros directivos del más alto nivel, así como egresados del Curso IGLU, procedentes de Canadá, Estados Unidos, México, Costa Rica, Puerto Rico, Colombia, Ecuador, Perú, Uruguay, Paraguay, Brasil y Argentina.

8 "Liderazgo Innovador: el gran desafío universitario (2011). Conferencia presentada en el Seminario de Gestión y Liderazgo Universitario. Xalapa, Ver. México, 14 de febrero. 


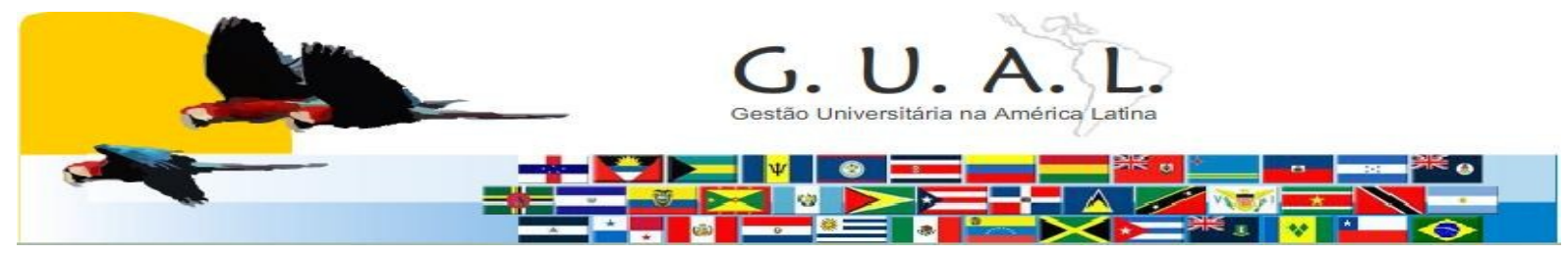

ISSN 1983-4535

\title{
MOVING WILLS: AGENTS OF CHANGE AND THE AMERICAN UNIVERSITY ORGANIZATION
}

\author{
Maria del Pilar Velasco Muñoz-Ledo, Master \\ Universidad Veracruzana - México \\ IGLU. OUI. \\ mvelasco@uv.mx
}

\begin{abstract}
In recent years, higher education has faced one of the most serious challenges of the last few decades, involving limitations and specific trials on the establishment of its agenda on a global level. Higher education institutions (HEI) face demands and pressure to fulfill their duty to a society that drastically transforms and that must deal with emerging issues and challenges that require new scientific, social and professional development to meet social needs. It would be erroneous to believe that the transformation of higher education only rests on the clarity of its purpose oriented to the future: the development of agents who will bring about change is also necessary. Academics are, without a doubt, fundamental actors in the design and preparation of this evolution, but to redefine the role of research, training and outreach also implies a change in organization and management. Therefore, HEI leaders and administrators must prepare to anticipate and direct the changes that will result in better institutions that make greater social contributions. The Inter-American Organization for Higher Education (IOHE) strives to contribute to the training of such agents, through the Institute of University Management and Leadership (IGLU) and the services it provides to the member institutions.
\end{abstract}

Keywords: Wills. Agents. University. 